ISSN: 2386-3919 - e-ISSN: 2386-3927

DOI: https://doi.org/10.14201/et2017351143160

\title{
ESTUDIO DE LA ELECCIÓN DEL ESPAÑOL COMO LENGUA EXTRANJERA EN LA ESCUELA SUPERIOR DE PORTALEGRE (PORTUGAL)
}

\section{Research on the choice of Spanish as a foreign language in the School of Portalegre (Portugal)}

\author{
C. SAntíño Grelo*, A. M. Rodríguez-García ${ }^{* *}$ y M. A. Hinojo LuCEnA**** \\ *Correo-e: corde@esep.pt \\ *arodrigu@ugr.es \\ ***marianbl@ugr.es
}

Recibido: 28/02/2017; Aceptado: 26/09/2017; Publicado: 31/05/2017

Ref. Bibl. C. SANTÍÑO GRELO, A. M. RODRÍGUEZ-GARCÍA y M. A. HINOJO LUCENA. Estudio de la elección del español como lengua extranjera en la Escuela Superior de Portalegre (Portugal). Enseñanza \& Teaching, 35, 1-2017, 143-160.

RESUMEN: El presente estudio es de gran relevancia para la práctica pedagógica al centrarse en las percepciones del alumnado portugués que decide optar por el aprendizaje del español como lengua extranjera, sobre las que se podrán realizar modificaciones didácticas y organizativas para adaptarse a las motivaciones diversas del aula. Así, ayudará a conocer e identificar los motivos y consideraciones que este idioma presenta, con el consiguiente replanteamiento de planes de estudios; asî como incorporando la posibilidad de trasladar este mismo estudio a otros contextos diferentes, tanto a nivel geográfico, como socioeconómico y cultural. De esta manera, el presente artículo pretende analizar las percepciones que explican el aumento progresivo del estudio de la lengua española por alumnos de Educación Superior en Portugal, concretamente a través de la Escuela Superior de Educación de Portalegre (ESEP), referente importante en la enseñanza del español como lengua extranjera. Para ello, se llevó a cabo una metodología descriptiva y mixta, en la que se integraron instrumentos cuantitativos (cuestionario escala tipo Likert) y cualitativos (entrevista semiestructurada), con un diseño no experimental por método de encuesta. Como 

DE PORTALEGRE (PORTUGAL)

principales resultados y aportaciones, encontramos que prácticamente la totalidad de quienes han tenido formación previa en español opinan que el español es más fácil que las demás lenguas, creen que pueden obtener mejores notas, trabajo más fácilmente y han elegido esta lengua porque van mucho a España. Así pues, la cercanía geográfica con España, las salidas profesionales, así como el origen románico del español, son señalados como los principales motivos para su elección.

Palabras clave: aprendizaje; español como lengua extranjera; educación superior; motivaciones; alumnado.

SUMMARY: This study is of great significance for teaching practice by focusing on perceptions of Portuguese students who decide to opt for learning Spanish as a foreign language, on which can be made educational and organizational changes to adapt to the different motivations of the classroom. So it helps you understand and identify the reasons and considerations that this language has, thereby rethinking curriculum; and incorporating the possibility of transferring this same study to other contexts, both geographically, and socio-economic and cultural. In this manner, the present paper aims to analyze the perceptions that explain the progressive increase of Spanish language study by students of higher education in Portugal, specifically, through on the School of Education of Portalegre (ESEP). So it was applied a descriptive and mixed methodology in which quantitative tools are integrated (Likert scale questionnaire) and qualitative (semi-structured interview), with a non-experimental design by survey method. As the main results and contributions, they were found that, practically the majority of students who has received a previous training of Spanish consider that Spanish is easier than other languages, they can get easily a good job and high marks and they have chosen this language because they go to Spain so much. Thus, the geographical proximity to Spain, as well as, the professional outputs and the Romanic origin of Spanish, are as the main reasons for their choice.

Key words: learning; Spanish as a foreign language; higher education; motivation; students.

\section{INTRODUCCIÓN}

El estudio del español como lengua extranjera se ha venido desarrollando desde hace tiempo por diferentes países y continentes, lo que ha supuesto el incremento de estudios centrados tanto en la caracterización de su enseñanza, como de su proceso de aprendizaje, dependiendo de la lengua materna inicial, adoptada como punto de partida (Sánchez, 2014; García, 2014; Bernardo, Amerigo y García, 2014; Flores-Ferrán y Lovejoy, 2015; Nakatsukasa y Loewen, 2015).

Sin embargo, la enseñanza del español como lengua extranjera en Portugal ha sido tradicionalmente minoritaria, en detrimento del francés, inglés o alemán, aunque los datos estadísticos oficiales de los últimos años muestran un aumento progresivo del estudio de la Lengua Española (Menéndez, 1980; Catalán, 1989; Baralo, 1999; Gómez, 2011; Santos, 2011; Tobar y Mañas, 2014). En este sentido, 
C. SANTÍÑO GRELO, A. M. RODRÍGUEZ-GARCÍA Y M. A. HINOJO LUCENA

ESTUDIO DE LA ELECCIÓN DEL ESPAÑOL COMO LENGUA EXTRANJERA EN LA ESCUELA SUPERIOR DE PORTALEGRE (PORTUGAL)

Ricardo (2012) y Martín (2012) señalaban que el incremento del alumnado que estudiaba español que se había producido en los últimos años había sido verdaderamente asombroso, convirtiéndose en la segunda lengua más solicitada del país en centros públicos y privados de enseñanza secundaria en Portugal, desbancando la posición que había ocupado el francés hasta entonces.

El caso de la Escuela Superior de Educación de Portalegre no es una excepción en lo que al interés por el estudio del español como lengua extranjera se refiere, ya que, según datos de la secretaría del centro, prácticamente el 90\% de los/ as alumnos/as que se matriculan cada año en la Escuela escogen el español como primera opción. Los motivos son muy variados, pero quizá el hecho de ser lenguas tan parecidas, lo cual se puede explicar por un origen común, o la cercanía de ambos países, toman gran importancia a la hora de decidirse por el español ante las otras opciones del centro (inglés, francés y alemán) (Martín, 2012). A su vez, otros autores destacan la cercanía entre ambos países y la atracción que España supone para los portugueses como razones importantes a la hora de comenzar los estudios de la lengua española (Rabadán, 2014).

Por otro lado, en lo que respecta a la comprensión de la lengua extranjera es favorecida cuando hay semejanza con la materna, tal y como señala Sweet (1964), al mencionar que existe facilidad en términos de comprensión cuando se trata de lenguas afines y cita, como ejemplo, los pares español-portugués, dinamarqués-sueco e inglés-escocés. De la misma forma, Takeuchi (1980) afirma que las lenguas portuguesa y española poseen un pequeño número de palabras diferentes y una gran variedad de vocablos comunes.

Cuando se trata de lenguas cognadas, como es el caso del portugués y el español, se sabe que la proximidad es un factor que favorece el aprendizaje. Según Almeida (1995), hasta las personas poco ansiosas son capaces de arriesgarse, pudiendo avanzar y adquirir eficiencia más rápidamente en una lengua próxima a la suya de lo que lo harían con aquellas tipológicamente más distintas, como el inglés. Así pues, las ventajas que la lengua materna ejerce en la comprensión de la lengua extranjera es, sin duda, un factor que fuertemente condiciona esa facilidad (Sola, Hinojo y Cáceres, 2010; Mira, 2012), sin olvidar el empleo de estrategias de enseñanza efectivas para el aprendizaje de una nueva lengua (Franco, Pino y Rodríguez, 2009), así como el uso de diferentes métodos didácticos encaminados hacia su consecución (Pierce y Cuadrado, 2011; Sevillano, 2011).

La proximidad tipológica del español y del portugués, con un tronco común y una historia evolutiva paralela, es una realidad percibida sin mayor dificultad por cualquier hablante nativo de cada una de las dos lenguas (Vigón, 2004; Vigón, Humblé y Vasconcellos, 2005; Vivanco, 2007; Vigón y Saracho, 2008; Aznar, Cáceres e Hinojo, 2008). La afinidad entre ambas lenguas es, por tanto, evidente. Ahora bien, tengamos en cuenta la advertencia que Dittami (1998) mencionó sobre el italiano respecto al español, diciendo que las apariencias, también en la lengua, pueden llegar a engañar y el origen neolatino de los dos idiomas los convierte, muy a menudo, en hermanos gemelos cuando sólo son familiares lejanos. 
C. SANTÍÑO GRELO, A. M. RODRÍGUEZ-GARCÍA Y M. A. HINOJO LUCENA

ESTUDIO DE LA ELECCIÓN DEL ESPAÑOL COMO LENGUA EXTRANJERA EN LA ESCUELA SUPERIOR DE PORTALEGRE (PORTUGAL)

Es fácil, para un hablante de portugués, entender el español escrito, pero no lo es tanto comprender el español hablado, expresarse en español (Campos, Sola y Aznar, 2014) -que no en portuñol- o traducir al español; es decir, tal y como indica Dittami (1998), dominar una lengua de manera precisa y gratificante es una tarea altamente complicada, más aún cuando hablamos de aprender y ser capaces de expresarnos en un idioma diferente al nuestro de forma rigurosa y precisa.

De este modo, vemos cómo distintos autores han tratado de explicar la vinculación existente entre lenguas consideradas similares (portugués-español, español-italiano, inglés-escocés, etc.). Sin embargo, son escasas las investigaciones centradas en describir empíricamente cuáles son las percepciones que los estudiantes portugueses tienen del español y qué motivos atribuyen para optar por su estudio como lengua extranjera. En este sentido, Martín (2012) señalaba una serie de motivaciones de índole política y económica que podían explicar tal fenómeno, tales como: la facilidad de acceso al mundo español, a su cultura, interacción con otras personas debido, fundamentalmente, a la proximidad entre ambos países; también destacaba la desaparición de las fronteras y la presencia de Portugal y España en la Comunidad Europea, así como el desarrollo empresarial que España había tenido en Portugal y viceversa.

Sin embargo, estas razones quedan aisladas si no parten de un contexto específico donde se lleve a cabo la investigación para conocer las razones que llevan al alumnado portugués a matricularse en español en lugar de otras lenguas. Entendemos que dichos motivos pueden ayudar a explicar, en cierta medida, la elección de esta lengua en el currículum pero, a su vez, se hace necesario conocer otras razones que llevan al estudiantado a su matriculación para continuar en el avance de la enseñanza del español como lengua extranjera en el contexto de Portugal.

\section{Metodología}

La presente investigación pertenece a otra de mayor envergadura y se caracteriza por ser de tipo exploratorio y no experimental, pues es el primer estudio que se realiza acerca de las motivaciones que llevan a los estudiantes de la muestra a elegir el español como primera lengua extranjera en Portalegre. Para ello seguiremos una metodología mixta o híbrida de investigación, combinando instrumentos de naturaleza cuantitativa (cuestionario) y cualitativa (entrevista). De esta manera se conseguirá una interpretación significativa y contextualizada de los datos obtenidos a partir del cuestionario mediante la posibilidad de profundizar en la realidad descrita por medio de la información facilitada a través de las entrevistas.

Así pues, el objetivo general de nuestra investigación ha sido:

- Analizar las motivaciones del alumnado de la Escuela Superior de Portalegre (Portugal) ante el estudio del español como lengua extranjera. 
C. SANTíÑO GRELO, A. M. RODRÍGUEZ-GARCÍA Y M. A. HINOJO LUCENA

ESTUDIO DE LA ELECCIÓN DEL ESPAÑOL COMO LENGUA EXTRANJERA EN LA ESCUELA SUPERIOR DE PORTALEGRE (PORTUGAL)

Por otro lado, en lo que respecta a los objetivos específicos, éstos han sido los siguientes:

- Examinar las motivaciones del alumnado de la ESEP ante el estudio del español como lengua extranjera en función del género.

- Estudiar las motivaciones del alumnado de la ESEP ante el estudio del español como lengua extranjera en función de la carrera.

- Explorar las motivaciones del alumnado de la ESEP ante el estudio del español como lengua extranjera en función de la formación previa.

Para dar respuesta a estos objetivos, en primer lugar, se realizó una revisión bibliográfica exhaustiva que nos llevó a percibir la escasa profundidad con la que se había abordado el tema, existiendo un vacío empírico que apoyase nuestro propósito. Por ello, consideramos que para conseguir una mayor profundización en el objetivo de estudio sería necesario proceder a indagar la realidad mediante el uso del cuestionario y de la entrevista semiestructurada.

\subsection{Instrumentos de recogida de datos}

El cuestionario fue diseñado "ad hoc» (Santíño, 2012), cumpliendo con las características psicométricas propias de este tipo de instrumentos, esto es, obteniendo una validez de contenido por juicio de expertos y un medio-alto coeficiente de fiabilidad Alfa de Cronbach de 0,872.

En su composición se ha seguido una escala tipo Likert de graduación de respuesta con un intervalo de 1-4 (totalmente en desacuerdo-totalmente de acuerdo), contabilizándose 91 variables en sus 9 bloques:

- Perfil personal y académico: nombre, edad, sexo, curso, carrera, formación previa...

- Motivaciones: se alude a las diferentes razones por las que se ha escogido el español como lengua extranjera, tales como la proximidad léxica entre ésta y el portugués, la cercanía entre ambos países, el gusto por la cultura española o por la creencia de consecución de una mejora laboral, entre otros.

- Finalidad: se señalan distintos tipos de fines del estudio de la lengua como la posibilidad de abrir horizontes hacia nuevos países, prosperar laboralmente, necesidades comunicativas por distintas razones (familiares, amigos, profesionales), etc.

- Percepciones sobre las destrezas: se señalan diferentes apreciaciones sobre el nivel de dificultad de la expresión oral, escrita y de comprensión.

- Técnicas de expresión escrita: se refiere a diferentes técnicas que les son más importantes y útiles para el aprendizaje de la lengua.

- Competencias comunicativas: se recogen diferentes percepciones sobre las habilidades de comunicación para el aprendizaje de la lengua. 

DE PORTALEGRE (PORTUGAL)

- Metodología: hace alusión a diferentes métodos empleados por el profesor para mejorar el aprendizaje del alumno.

- Actividades y recursos didácticos: engloba ítems referentes a las diferentes acciones y recursos empleados para que el aprendizaje se produzca con mayor eficacia.

- Organización: esta dimensión menciona ítems referentes al horario, al número de alumnos por profesor, distribución de contenido teórico y práctico, etc.

En lo que respecta a la construcción de la entrevista, ésta fue realizada de manera semiestructurada atendiendo a las mismas dimensiones anteriormente citadas (perfil personal y académico; motivaciones; adquisición de la lengua; percepción sobre las destrezas; técnicas de expresión escrita; competencias comunicativas; metodología; actividades y recursos didácticos; y organización), pero que dejan un poco más abierta la respuesta para obtener información que el cuestionario por sí solo no puede recabar.

Atendiendo más concretamente a los objetivos de investigación, el bloque referido al aspecto "motivaciones", éste se compone de una única pregunta en la entrevista que dice así: ¿cuál fue el motivo que te llevó a escoger español en lugar de otras lenguas como francés, inglés o alemán? En lo que respecta al cuestionario, esta dimensión está compuesta por 12 ítems referentes a diferentes aspectos acerca de las motivaciones que llevan al alumnado portugués a elegir el español como lengua extranjera.

\subsection{Población y muestra}

El universo o población se corresponde con los estudiantes pertenecientes a la Escuela Superior de Educación de Portalegre (ESEP) que tienen el español entre sus unidades curriculares. Por lo tanto, serían todos los estudiantes de la carrera de Periodismo y Comunicación, Animación Sociocultural, Servicio Social y Turismo de la Escuela Superior de Educación de Portalegre que cursan el español como lengua extranjera (82 alumnos).

De este grupo se ha extraído una muestra representativa a efectos cuantitativos y estadísticos de la población a la que pertenece. La elección de la muestra ha sido aleatoria representativa con un nivel de confianza del $95 \%$ y un intervalo de confianza del $8 \%$. Más concretamente, y al tratarse de instrumentos de distinta índole, la muestra estuvo compuesta por $\mathrm{n}=53$ participantes para el cuestionario y de $n=15$ para las entrevistas pues, en este último caso, no se pretende generalizar a la población, sino descubrir razones personales que aporten datos al objeto de estudio.

La recogida de datos se realizó en dos cursos diferentes y la muestra queda reflejada en la siguiente tabla: 
C. SANTÍÑ̃ GRELO, A. M. RODRÍGUEZ-GARCÍA Y M. A. HINOJO LUCENA

ESTUDIO DE LA ELECCIÓN DEL ESPAÑOL COMO LENGUA EXTRANJERA EN LA ESCUELA SUPERIOR DE PORTALEGRE (PORTUGAL)

TABLA 1

Distribución de la muestra por carrera y curso académico

\begin{tabular}{|c|c|c|c|c|c|c|}
\cline { 3 - 7 } \multicolumn{2}{c|}{} & $\begin{array}{c}\text { ANIMACIÓN } \\
\text { SOCIOCUltural }\end{array}$ & $\begin{array}{c}\text { PERIODISMO Y } \\
\text { COMUNICACIÓN }\end{array}$ & $\begin{array}{c}\text { SERVICIOS } \\
\text { SOCIALES }\end{array}$ & TURISMO & \\
\hline \multirow{3}{*}{$2014 / 2015$} & Primero & 0 & 8 & 0 & 11 & \\
\cline { 2 - 8 } & Segundo & 0 & 0 & 0 & 7 & \\
\cline { 2 - 8 } & Total & - & 8 & - & 18 & 26 \\
\hline $2015 / 2016$ & Primero & 4 & 6 & 4 & 13 & 27 \\
\hline \multicolumn{2}{|c|}{ Total } & 4 & 14 & 4 & 31 & 53 \\
\hline
\end{tabular}

FUENTE: Elaboración propia

De este modo, un total de 53 alumnos respondieron a nuestra encuesta -lo que representa un 64,63\% de la población-, con edades comprendidas entre los 17 y los 53 años, y una media de edad que se sitúa entre los 18 y los 21 años. Habiendo un 20,8\% de 19 años, un $17 \%$ de 20 y un $15 \%$ de 21, el mismo número que de 18.

En relación al sexo, 37 de los 53 participantes, es decir, casi el 70\%, son del sexo femenino. Los 16 alumnos restantes, el 30,2\%, pertenecen al sexo masculino.

Por otro lado, el 86,8\% son alumnos de primer año, y el 13,2\% de segundo año. En relación a la carrera, el 58,49\%, son alumnos de Turismo, 26,42\% de Periodismo y Comunicación, el 7,55\% de Servicio Social y el 7,55\% restante de Animación Sociocultural.

De los alumnos participantes, una gran mayoría, el 90,6\%, no ha estudiado español antes de llegar a la ESEP, lo que vendría a avalar el hecho de que, pese al aumento del estudio de esta lengua en secundaria, aún no ha llegado a alcanzar los niveles de otras lenguas extranjeras como el inglés, por ejemplo.

\subsection{Análisis de datos}

Para el análisis de los datos cuantitativos, se procedió al tratamiento y análisis estadístico de los cuestionarios mediante el paquete estadístico sPss versión 22.0 para Windows. El proceso ha consistido en importar todos los datos, etiquetarlos y posteriormente se ha realizado un análisis descriptivo.

Respecto al instrumento cualitativo, el procedimiento consistió en la codificación y categorización de la información obtenida a través de la entrevista con ayuda del software Atlas.ti. 

DE PORTALEGRE (PORTUGAL)

\section{Resultados}

Para la obtención de los resultados se han llevado a cabo análisis estadísticos descriptivos, tales como análisis de frecuencias, y análisis inferencial a través de pruebas no parámetricas [chi-cuadrado de Pearson con sus respectivos grados de libertad (gl) y significatividad asincrónica]. Para la parte de las entrevistas, se ha seguido un proceso de transcripción, codificación y categorización, estableciendo nodos relacionales e interconectados entre sí. A continuación se presentan los resultados más sugerentes obtenidos atendiendo a nuestros objetivos de investigación.

En primer lugar, se presenta un gráfico donde podemos observar los diferentes ítems que han compuesto la dimensión de "motivación" con sus respuestas pertinentes en cada uno de ellos. De este modo, los dos principales motivos que llevan al alumnado a decidirse por el estudio del español es el estar cerca de España y que es una lengua importante para su futuro profesional, en ambos casos hay un $84,9 \%$ de los alumnos que dice estar de acuerdo con que este es el motivo por el que decidieron estudiar español. Muy de cerca, un 83\% de alumnos se encuentran de acuerdo con la afirmación de que «son lenguas parecidas» y que «es útil para establecer contactos comerciales». El motivo que parece ser el que menos ha movido a los alumnos a la hora de elegir el español es "Voy mucho a España", con un $54,7 \%$ de los alumnos que están de acuerdo. En lugares intermedios estaría las siguientes motivaciones: "me atrae la cultura española", con 69,8\%; "creo que puedo obtener mejores notas" y "puedo encontrar trabajo más fácilmente», ambas con 67,9\%; «es una lengua que me apasiona», con 64,2\%; "es más fácil que las demás»y "es una lengua nueva para mí y quería conocerla», ambas con 62,3\%; y "España y lo español están de moda", con 60,3\%.

En segundo lugar, en lo que respecta a las respuestas obtenidas mediante las entrevistas, en la siguiente tabla se recogen las motivaciones más señaladas por los 15 alumnos entrevistados y que nos arrojan un mayor nivel de profundidad sobre las razones que les han llevado a elegir el español como lengua extranjera.

En tercer lugar, se presentan los datos significativos obtenidos a partir de las tablas de contingencia y que representan los cruces realizados entre las variables y estadísticos descriptivos de nuestro estudio. Hemos de afirmar la no existencia de diferencias significativas entre el sexo y las motivaciones para el estudio del español. Solamente se aprecian diferencias significativas en dos ítems: «Decidí estudiar español porque España y lo español están de moda", mostrando un menor acuerdo por parte de las mujeres (32,5\%) frente a los hombres (50\%). Al contrario ocurre con la afirmación: «Decidí estudiar español porque es una lengua nueva para mí y quería conocerla»; en este caso, son las mujeres $(67,6 \%)$ quienes se posicionan más afines a este ítem que los hombres (50\%). 
C. SANTÍÑO GRELO, A. M. RODRÍGUEZ-GARCÍA Y M. A. HINOJO LUCENA

ESTUDIO DE LA ELECCIÓN DEL ESPAÑOL COMO LENGUA EXTRANJERA EN LA ESCUELA SUPERIOR DE PORTALEGRE (PORTUGAL)

FigURA 1

Resumen de los resultados obtenidos en relación a las motivaciones de los alumnos de la ESEP para estudiar español

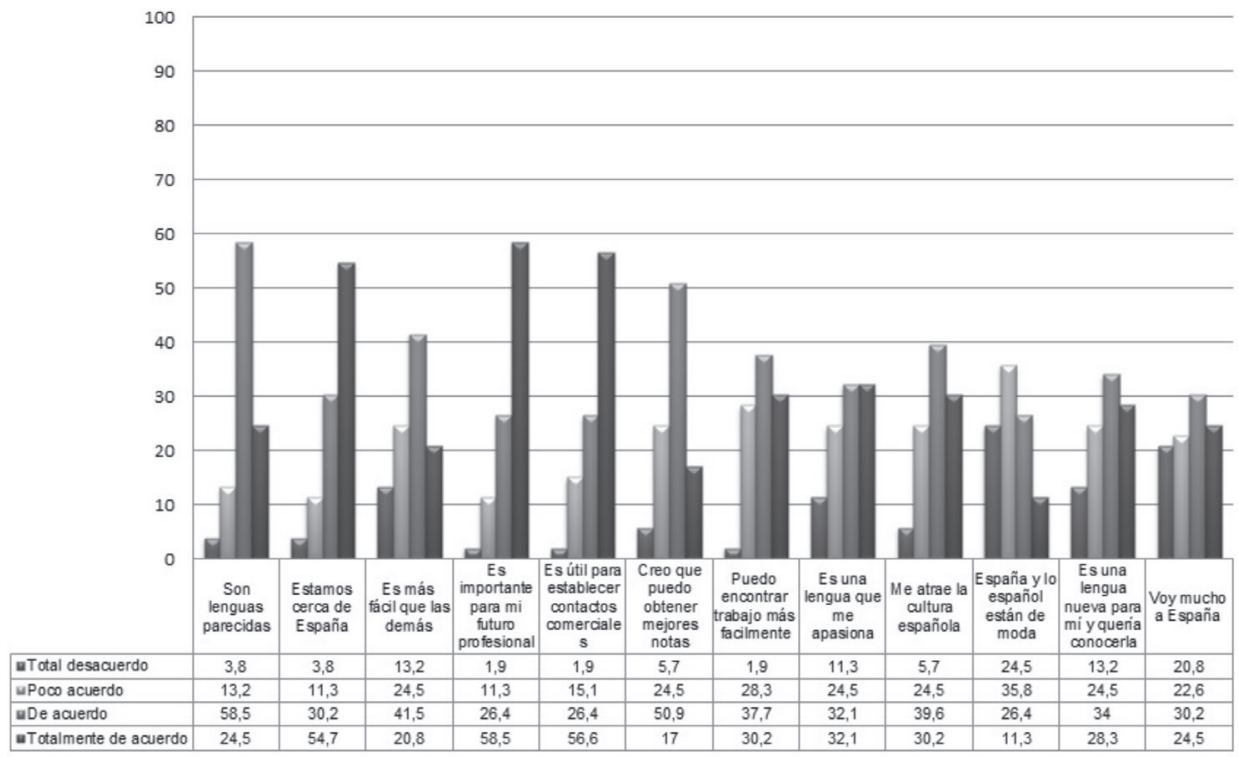

FUENTE: Elaboración propia.

TABLA 2

Codificación de los resultados de entrevistas

\begin{tabular}{|l|l|}
\cline { 2 - 3 } \multicolumn{1}{c|}{} & \multicolumn{1}{c|}{ MotrvaCiONES } \\
\hline Entrevista 1 & - Ya ha tenido español previamente y le despertaba bastante interés. \\
\hline Entrevista 2 & $\begin{array}{l}- \text { Ya conocía las otras lenguas. Le gusta España, tiene } \\
\text { amigos allí y le gustaría vivir allí y hacer Erasmus allí. }\end{array}$ \\
\hline Entrevista 3 & $\begin{array}{l}\text { Nunca ha estudiado español y tenía curiosidad por conocer } \\
\text { la lengua, también piensa ir de Erasmus a España. }\end{array}$ \\
\hline Entrevista 4 & - Le puede ayudar a conseguir un trabajo en España. \\
\hline Entrevista 5 & $\begin{array}{l}\text { El español es importante y es una de las } \\
\text { lenguas más habladas del mundo. }\end{array}$ \\
\hline Entrevista 6 & - El español es una de las lenguas más habladas en el mundo. \\
\hline Entrevista 7 & - Por conocer una lengua nueva. \\
\hline Entrevista 8 & - Tiene facilidad para el español y también tiene familia en España. \\
\hline Entrevista 9 & $\begin{array}{l}\text { El español es una lengua que hoy hace mucha falta, es una de las } \\
\text { lenguas más habladas y puede ayudar a conseguir trabajo en España. }\end{array}$ \\
\hline
\end{tabular}



DE PORTALEGRE (PORTUGAL)

\begin{tabular}{|l|l|}
\cline { 2 - 3 } \multicolumn{1}{c|}{} & \multicolumn{1}{c|}{ MotivaCıONES } \\
\hline Entrevista 10 & - La lengua es parecida y hay más posibilidades de ir a España. \\
\hline Entrevista 11 & - Es más fácil que las otras lenguas. \\
\hline Entrevista 12 & - Es más fácil que las otras lenguas, «ya tuve francés y suspendí». \\
\hline Entrevista 13 & $\begin{array}{l}\text { Es una lengua activa en Portugal que está } \\
\text { siendo «invadido» por españoles. }\end{array}$ \\
\hline Entrevista 14 & - Es una lengua que se está desarrollando y es más fácil que las otras. \\
\hline Entrevista 15 & - Es más fácil que las otras. \\
\hline
\end{tabular}

FUENTE: Elaboración propia.

TABLAS 3 y 4

Estadísticos descriptivos y prueba de chi-cuadrado en el ítem «Decidí estudiar español porque España y lo español están de moda»

\begin{tabular}{|c|c|c|c|c|c|c|}
\hline & \multicolumn{5}{|c|}{ DECIDÍ ESTUDIAR ESPAÑOL PORQUE ESPAÑA Y LO ESPAÑOL ESTÁN DE MODA } & \multirow[b]{2}{*}{ TOTAL } \\
\hline & $\begin{array}{c}\text { TOTALMENTE EN } \\
\text { DESACUERDO }\end{array}$ & $\begin{array}{l}\text { POCO DE } \\
\text { ACUERDO }\end{array}$ & $\begin{array}{c}\text { DE } \\
\text { ACUERDO }\end{array}$ & $\begin{array}{l}\text { TOTALMENTE } \\
\text { DE ACUERDO }\end{array}$ & $\mathrm{NS} / \mathrm{NC}$ & \\
\hline Hombre & $18,8 \%$ & $25 \%$ & $37,5 \%$ & $12,5 \%$ & $6,2 \%$ & 16 \\
\hline Mujer & $27 \%$ & $40,5 \%$ & $21,7 \%$ & $10,8 \%$ & $0 \%$ & 37 \\
\hline Total & $24,5 \%$ & $35,8 \%$ & $26,5 \%$ & $11,3 \%$ & $1,9 \%$ & 53 \\
\hline
\end{tabular}

Chi-cuadrado de Pearson

\begin{tabular}{|c|c|c|}
\hline VALOR & GL & Sig. ASINTÓNICA (BILATERAL) \\
\hline $4,471^{\mathrm{a}}$ & 4 &, 346 \\
\hline
\end{tabular}

A. 6 casillas $(60,0 \%)$ tienen una frecuencia esperada inferior a 5. La frecuencia mínima esperada es ,30. FuENTE: Elaboración propia.

En cuarto lugar, si atendemos a las diferentes motivaciones en función de la carrera encontramos que todos los alumnos de Servicio Social y más de la mitad del alumnado de Periodismo (57,2\%) y Turismo (64,5\%) señalan esta opción con un grado de acuerdo alto o muy alto, a diferencia del alumnado de Animación Sociocultural (25\%). A continuación, en las tablas 7-10 presentamos los estadísticos descriptivos y prueba de chi-cuadrado que se han obtenido en relación a diferentes motivaciones (mejores notas y posibilidades laborales) en función de la carrera.

En este sentido observamos que más de la mitad de los alumnos de las diferentes carreras se encuentran con un grado de acuerdo alto o muy alto con la motivación de sacar mejores notas para la elección del español como lengua extranjera. Destaca el alumnado de Animación Sociocultural (75\%), Periodismo $(71,4 \%)$, Servicio Social $(67,9 \%)$ y Turismo $(67,7 \%)$. 
C. SANTÍÑ̃ GRELO, A. M. RODRÍGUEZ-GARCÍA Y M. A. HINOJO LUCENA

ESTUDIO DE LA ELECCIÓN DEL ESPAÑOL COMO LENGUA EXTRANJERA EN LA ESCUELA SUPERIOR DE PORTALEGRE (PORTUGAL)

\section{TABLAS 5 y 6}

Estadísticos descriptivos y prueba de chi-cuadrado en el ítem «Decidí estudiar español porque es una lengua nueva para mí y quería conocerla»

\begin{tabular}{|c|c|c|c|c|c|c|}
\hline & \multicolumn{5}{|c|}{ DECIDÍ ESTUDIAR ESPAÑOL PORQUE ESPAÑA Y LO ESPAÑOL ESTÁN DE MODA } & \multirow[b]{2}{*}{ TOTAL } \\
\hline & $\begin{array}{c}\text { TOTALMENTE EN } \\
\text { DESACUERDO }\end{array}$ & $\begin{array}{l}\text { POCO DE } \\
\text { ACUERDO }\end{array}$ & $\begin{array}{c}\text { DE } \\
\text { ACUERDO }\end{array}$ & $\begin{array}{l}\text { TOTALMENTE } \\
\text { DE ACUERDO }\end{array}$ & $\mathrm{NS} / \mathrm{NC}$ & \\
\hline Hombre & $18,8 \%$ & $31,2 \%$ & $12,5 \%$ & $37,5 \%$ & $6,2 \%$ & 16 \\
\hline Mujer & $10,8 \%$ & $21,6 \%$ & $43,3 \%$ & $24,3 \%$ & $0 \%$ & 37 \\
\hline Total & $13,2 \%$ & $24,5 \%$ & $34 \%$ & $28,3 \%$ & $1,9 \%$ & 53 \\
\hline
\end{tabular}

Chi-cuadrado de Pearson

\begin{tabular}{|c|c|c|}
\hline VALOR & GL & Sig. ASINTÓNICA (BILATERAL) \\
\hline $4,74^{\mathrm{a}}$ & 3 &, 191 \\
\hline
\end{tabular}

A. 4 casillas $(50,0 \%)$ tienen una frecuencia esperada inferior a 5 . La frecuencia mínima esperada es 2,11. FUENTE: Elaboración propia.

TABLAS 7 y 8

Estadísticos descriptivos y prueba de chi-cuadrado en el ítem «Decidí estudiar español porque creo que puedo obtener mejores notas»

\begin{tabular}{|c|c|c|c|c|c|c|}
\cline { 2 - 7 } \multicolumn{1}{c|}{} & \multicolumn{4}{c|}{$\begin{array}{r}\text { DECIDÍ ESTUDIAR ESPAÑOL PORQUE CREO QUE } \\
\text { PUEDO OBTENER MEJORES NOTAS }\end{array}$} & \multirow{2}{*}{ TOTAL } \\
\cline { 2 - 7 } & $\begin{array}{c}\text { TOTALMENTE } \\
\text { EN } \\
\text { DESACUERDO }\end{array}$ & $\begin{array}{c}\text { POCO DE } \\
\text { ACUERDO }\end{array}$ & $\begin{array}{c}\text { DE } \\
\text { ACUERDO }\end{array}$ & $\begin{array}{c}\text { TOTALMENTE } \\
\text { DE ACUERDO }\end{array}$ & NS/NC & \\
\hline Periodismo & $7,1 \%$ & $14,3 \%$ & $50 \%$ & $21,4 \%$ & $7,1 \%$ & 14 \\
\hline Turismo & $3,2 \%$ & $29 \%$ & $54,8 \%$ & $12,9 \%$ & $0 \%$ & 31 \\
\hline $\begin{array}{c}\text { Animación } \\
\text { Sociocultural }\end{array}$ & $25 \%$ & $0 \%$ & $50 \%$ & $25 \%$ & $0 \%$ & 4 \\
\hline Servicio Social & $0 \%$ & $50 \%$ & $25 \%$ & $25 \%$ & $0 \%$ & 4 \\
\hline Total & $5,7 \%$ & $24,5 \%$ & $50,9 \%$ & $17 \%$ & $1,9 \%$ & 53 \\
\hline
\end{tabular}

\begin{tabular}{|c|c|c|c|}
\cline { 2 - 4 } \multicolumn{1}{c|}{} & VALOR & GL & SIG. ASINTÓNICA (BILATERAL) \\
\hline Chi-cuadrado de Pearson & $10,322^{\mathrm{a}}$ & 12 &, 588 \\
\hline
\end{tabular}

A. 16 casillas $(80,0 \%)$ tienen una frecuencia esperada inferior a 5 . La frecuencia mínima esperada es ,08. FUENTE: Elaboración propia. 
C. SANTÍÑ̃O GRELO, A. M. RODRÍGUEZ-GARCÍA Y M. A. HINOJO LUCENA

ESTUDIO DE LA ELECCIÓN DEL ESPAÑOL COMO LENGUA EXTRANJERA EN LA ESCUELA SUPERIOR DE PORTALEGRE (PORTUGAL)

En función de la mayor facilidad para encontrar trabajo y la carrera de procedencia, todo el alumnado de Animación Sociocultural piensa que encontrarán trabajo más fácilmente si aprenden español, seguido del $71 \%$ de los alumnos de Turismo, el 57,2\% de Periodismo y, finalmente, el 50\% de Servicio Social.

TABLAS 9 y 10

Estadísticos descriptivos y prueba de chi-cuadrado en el ítem «Decidí estudiar español porque puedo encontrar trabajo más fácilmente»

\begin{tabular}{|c|c|c|c|c|c|c|}
\cline { 2 - 7 } \multicolumn{1}{c|}{} & \multicolumn{5}{c|}{$\begin{array}{c}\text { DECIDÍ ESTUDIAR ESPAÑOL PORQUE PUEDO } \\
\text { ENCONTRAR TRABAJO MÁS FÁCILMENTE }\end{array}$} & \multirow{2}{*}{ TOTAL } \\
\cline { 2 - 7 } & $\begin{array}{c}\text { TOTALMENTE } \\
\text { EN } \\
\text { DESACUERDO }\end{array}$ & $\begin{array}{c}\text { POCO DE } \\
\text { ACUERDO }\end{array}$ & $\begin{array}{c}\text { DE } \\
\text { ACUERDO }\end{array}$ & $\begin{array}{c}\text { TOTALMENTE } \\
\text { DE ACUERDO }\end{array}$ & NS/NC & \\
\hline Periodismo & $7,1 \%$ & $28,6 \%$ & $28,6 \%$ & $28,6 \%$ & $7,1 \%$ & 14 \\
\hline Turismo & $0 \%$ & $29 \%$ & $45,2 \%$ & $25,8 \%$ & $0 \%$ & 31 \\
\hline $\begin{array}{c}\text { Animación } \\
\text { Sociocultural }\end{array}$ & $0 \%$ & $0 \%$ & $25 \%$ & $75 \%$ & $0 \%$ & 4 \\
\hline Servicio Social & $0 \%$ & $50 \%$ & $25 \%$ & $25 \%$ & $0 \%$ & 4 \\
\hline Total & $1,9 \%$ & $28,3 \%$ & $37,7 \%$ & $30,2 \%$ & $1,9 \%$ & 53 \\
\hline
\end{tabular}

\begin{tabular}{|c|c|c|c|}
\cline { 2 - 4 } \multicolumn{1}{c|}{} & VALOR & GL & Sig. AsINTÓNICA (BILATERAL) \\
\hline Chi-cuadrado de Pearson & $11,389^{\mathrm{a}}$ & 12 &, 496 \\
\hline
\end{tabular}

A. 16 casillas $(80,0 \%)$ tienen una frecuencia esperada inferior a 5. La frecuencia mínima esperada es ,08. FUENTE: Elaboración propia.

En quinto lugar, si cruzamos la variable referente a la formación previa con los ítems analizados, encontramos que la gran mayoría de los alumnos que poseen formación previa en español se encuentran de acuerdo o totalmente de acuerdo con todas las afirmaciones presentadas (el español es más fácil que otras lenguas, mejores notas en español, trabajo más fácilmente y cantidad de veces que van a España), a diferencia de dos ítems, que son los referentes a la elección del español a causa de ser una nueva lengua para el sujeto y al que señala que España y lo español están de moda, lo que nos hace pensar que la formación previa es un factor clave en la motivación para la elección de esta lengua. A continuación, en las Tablas 11-14 se presenta el cruce de dos ítems: «Decidí estudiar español porque es una lengua nueva para mí y quería conocerla» $\mathrm{y}$ "Decidí estudiar español porque es más fácil que las demás» en función de la formación previa. 
C. SANTÍÑO GRELO, A. M. RODRÍGUEZ-GARCÍA Y M. A. HINOJO LUCENA

ESTUDIO DE LA ELECCIÓN DEL ESPAÑOL COMO LENGUA EXTRANJERA EN LA ESCUELA SUPERIOR DE PORTALEGRE (PORTUGAL)

\section{TABLAS 11 y 12}

Estadísticos descriptivos y prueba de chi-cuadrado en el ítem «Decidí estudiar español porque es una lengua nueva para mí y quería conocerla»

\begin{tabular}{|c|c|c|c|c|c|}
\cline { 2 - 5 } \multicolumn{1}{c|}{} & \multicolumn{4}{c|}{$\begin{array}{c}\text { DECIDÍ ESTUDIAR ESPAÑOL PORQUE ES UNA LENGUA } \\
\text { NUEVA PARA MÍ Y QUERÍA CONOCERLA }\end{array}$} & \multirow{2}{*}{ Total } \\
\cline { 2 - 5 } & $\begin{array}{c}\text { TotALMENTE EN } \\
\text { DESACUERDO }\end{array}$ & $\begin{array}{c}\text { POCO DE } \\
\text { ACUERDO }\end{array}$ & $\begin{array}{c}\text { DE } \\
\text { ACUERDO }\end{array}$ & $\begin{array}{c}\text { TOTALMENTE } \\
\text { DE ACUERDO }\end{array}$ & \\
\hline $\begin{array}{c}\text { Con formación } \\
\text { previa }\end{array}$ & $40 \%$ & $60 \%$ & $0 \%$ & $0 \%$ & 5 \\
\hline $\begin{array}{c}\text { Sin formación } \\
\text { previa }\end{array}$ & $10,4 \%$ & $20,8 \%$ & $37,5 \%$ & $31,3 \%$ & 48 \\
\hline Total & $13,2 \%$ & $24,5 \%$ & $34 \%$ & $28,3 \%$ & 53 \\
\hline
\end{tabular}

\begin{tabular}{|c|c|c|c|}
\cline { 2 - 4 } \multicolumn{1}{c|}{} & VALOR & GL & SIG. ASINTÓNICA (BILATERAL) \\
\hline Chi-cuadrado de Pearson & $9,270^{\mathrm{a}}$ & 3 &, 026 \\
\hline
\end{tabular}

A. 4 casillas $(50,0 \%)$ tienen una frecuencia esperada inferior a 5. La frecuencia mínima esperada es ,66 FUENTE: Elaboración propia.

TABLA 13 y 14

Estadísticos descriptivos y prueba de chi-cuadrado «Decidí estudiar español porque es más fácil que las demás»

\begin{tabular}{|c|c|c|c|c|c|}
\hline & \multicolumn{4}{|c|}{ DECIDÍ ESTUDIAR ESPAÑOL PORQUE ES MÁS FÁCIL QUE LAS DEMÁS } & \multirow[b]{2}{*}{ TOTAL } \\
\hline & $\begin{array}{c}\text { TOTALMENTE EN } \\
\text { DESACUERDO }\end{array}$ & $\begin{array}{l}\text { POCO DE } \\
\text { ACUERDO }\end{array}$ & $\begin{array}{c}\text { DE } \\
\text { ACUERDO }\end{array}$ & $\begin{array}{l}\text { TOTALMENTE } \\
\text { DE ACUERDO }\end{array}$ & \\
\hline $\begin{array}{c}\text { Con formación } \\
\text { previa }\end{array}$ & $0 \%$ & $0 \%$ & $40 \%$ & $60 \%$ & 5 \\
\hline $\begin{array}{c}\text { Sin formación } \\
\text { previa }\end{array}$ & $14,6 \%$ & $27,1 \%$ & $41,7 \%$ & $16,6 \%$ & 48 \\
\hline Total & $13,2 \%$ & $24,5 \%$ & $41,5 \%$ & $20,8 \%$ & 53 \\
\hline
\end{tabular}

\begin{tabular}{|c|c|c|c|}
\cline { 2 - 4 } \multicolumn{1}{c|}{} & VALOR & GL & Sig. ASINTÓNICA (BILATERAL) \\
\hline Chi-cuadrado de Pearson & $6,183^{\mathrm{a}}$ & 3 &, 103 \\
\hline
\end{tabular}

A. 4 casillas $(50,0 \%)$ tienen una frecuencia esperada inferior a 5. La frecuencia mínima esperada es ,66 FUENTE: Elaboración propia.

Como era de esperar, el 100\% de los alumnos con formación previa en español no ven esta afirmación como una motivación para escoger el español como lengua extranjera, a diferencia de aquellos alumnos que no poseían conocimientos 

DE PORTALEGRE (PORTUGAL)

sobre la lengua anteriormente puntuando, de esta forma, con un $68,8 \%$ de acuerdo o gran acuerdo con tal motivación.

Al igual ocurre con la consideración del español como una lengua más fácil con las demás. En este ítem, todo el alumnado con formación previa se encuentra de acuerdo con tal afirmación para la elección del español como lengua extranjera. Más dispersados se encuentran los valores dados por el alumnado sin formación previa: el 58,3\% de acuerdo o muy de acuerdo y el $41,7 \%$ restante se encuentra poco de acuerdo o muy en desacuerdo.

\section{CONCLUSIONES}

Antes de proceder a la conclusión y discusión de los resultados, es preciso detenernos para anunciar al lector acerca de la necesidad de tratar los datos con cierta cautela pues, como ya bien hemos indicado, se ha detectado cierto vacío empírico que se torna palpable en la línea de esta investigación, siendo ésta la primera planteada en el contexto de Portalegre. Sin embargo, sí constituye el presente estudio una base sólida para continuar avanzando en este campo indagando acerca de cómo mejorar la enseñanza del español en Portugal partiendo de la base de las motivaciones que llevan al alumnado a su matriculación en esta asignatura.

En primer lugar, sería conveniente realizar un esbozo general acerca de las diferentes motivaciones presentadas para la elección del español como lengua extranjera. En este sentido, ante la afirmación «Decidí estudiar español porque ambas son lenguas parecidas", más del 80\% del alumnado se encuentra de acuerdo o muy de acuerdo con esta afirmación. No cabe duda de que para un hablante de portugués el español presenta mucha más facilidad en comparación con otras lenguas como el inglés, el francés o el alemán (Ricardo, 2012), que son idiomas que actualmente tienen en la escuela como alternativa al español.

De manera similar, en el ítem "Decidí estudiar español porque estamos cerca de España», casi el 85\% está de acuerdo o muy de acuerdo con esta afirmación. Este resultado puede explicarse por el hecho de la cercanía entre España, Portalegre y Portugal, lo que implica, a su vez, que la posibilidad de mantener relaciones con este país sea mayor que con otros (Martín, 2012).

Por otro lado, el $41,5 \%$ de los participantes afirma estar de acuerdo con la afirmación "Decidí estudiar español porque es más fácil que las demás» y un 20,8\% afirma estar totalmente de acuerdo. Siendo así, el 62,3\% de la muestra indica que éste es uno de los motivos que le llevó a escoger el español.

Un alto porcentaje, el 76,5\%, afirma que ha escogido español porque esta lengua es importante para su futuro profesional. A su vez, más del 80\% está de acuerdo con la afirmación "Decidí estudiar español porque es útil para establecer contactos comerciales», resultado esperable si tenemos en cuenta la cercanía con España (Tobar y Mañas, 2014). 
C. SANTÍÑO GRELO, A. M. RODRÍGUEZ-GARCÍA Y M. A. HINOJO LUCENA

ESTUDIO DE LA ELECCIÓN DEL ESPAÑOL COMO LENGUA EXTRANJERA EN LA ESCUELA SUPERIOR DE PORTALEGRE (PORTUGAL)

Otro de los posibles motivos que llevan a los alumnos a escoger español es el hecho de poder obtener mejores notas. En este caso, casi el 70\% afirma que éste es uno de los motivos por los que escogieron español. Esta respuesta se relacionaría con la que dieron en relación a "Decidí estudiar español porque es más fácil que las demás», en la que alrededor del 62\% decía estar de acuerdo.

Hay también quien se decide por el español por considerar una mejora para su futuro laboral. En este sentido, casi el 70\% está de acuerdo con esta afirmación. Una vez más la cercanía con España tiene mucho que ver en este resultado pues sería más fácil para ellos desplazarse a España que a cualquier otro país. También por el deseo de conocer una lengua nueva con un $62 \%$ del alumnado.

El 64\% de los alumnos ha decidido estudiar español porque es una lengua que les apasiona y el 70\% porque les atrae la cultura española. Resultados similares a todos los anteriores ha obtenido la investigación de Rabadán (2014). Esta autora señala la predisposición positiva de los portugueses hacia todo aquello que tiene que ver con el país vecino, su cultura y su lengua.

En menor medida, las afirmaciones "España y lo español están de moda» y "Voy mucho a España" han obtenido puntuaciones más dispersas, siendo éstas las razones que menor mueven al alumnado a escoger el español como lengua extranjera.

En segundo lugar, en lo que respecta al sexo no se han encontrado diferencias estadísticas significativas que nos aporten datos de las motivaciones para la elección del español en función de ser hombre o mujer. Por ejemplo, ambos sexos afirman estudiar español porque ambas lenguas son parecidas (86\% de las mujeres, frente al $75 \%$ de los hombres), pueden sacar mejores notas (68,8\% los hombres y $67,2 \%$ las mujeres) siendo éstos los motivos más comúnmente seleccionados por ambos y obteniendo en el resto porcentajes similares, por lo que no existe correlación ninguna.

En tercer lugar, atendiendo a las motivaciones de elección del español en función de la carrera que se esté cursando tampoco se han encontrado resultados muy significativos que difieran de unos estudios a otros. Así pues, la gran mayoría del alumnado de las distintas carreras ha señalado que uno de los motivos más importantes a la hora de elegir el español como lengua extranjera es el hecho de que les resulte más fácil que las demás, al igual que con el hecho de obtener mejores notas o encontrar trabajo más fácilmente.

En cuarto lugar, en función de la formación previa del estudiando como motivo determinante en la elección del español como lengua extranjera se han encontrado diferencias significativas entre ambos grupos. Así pues, era de esperar que, para el estudiantado con experiencia en el idioma, la facilidad del mismo y la obtención de mejores notas hayan sido dos motivaciones principales para elegirla. En líneas generales, estos resultados son afines a los presentados por Rabadán (2014). Los conocimientos previos, a su vez, marcan un aspecto determinante en la apertura hacia una mejora laboral y el hecho de mantener más visitas y contactos con el país vecino. Quien ya conoce la lengua es consciente de que, pese a la dificultad que puede presentar la tarea de aprender un idioma extranjero, el hecho de 

DE PORTALEGRE (PORTUGAL)

ser una lengua románica, al igual que el portugués -su lengua materna-, conlleva que para ellos sea mucho más factible aprenderla debido a ciertas similitudes entre ambas, lo que facilitaría la adquisición de competencias comunicativas. En este sentido, un hablante de portugués es capaz entender, por lo menos de una forma general, un texto escrito o una comunicación oral en español, por lo que resulta mucho más fácil para él aprender español que otra lengua como, por ejemplo, el alemán. Esto le llevaría claramente a poder obtener mejores notas. Todo ello nos hace pensar que la formación es un elemento determinante en la respuesta de los sujetos, mostrándose aquellos que ya habían conocido el español con anterioridad más predispuestos al avance en el desarrollo del nivel del mismo.

Por otro lado, en lo que respecta a los resultados obtenidos a través de las entrevistas, éstos han servido para arrojar cierta luz donde el cuestionario solo hace sombras. En este sentido, han ayudado a profundizar en la cuestión y a señalar otras motivaciones para elegir el español en lugar de otra lengua. Así pues, vemos que las motivaciones de los alumnos encuestados para estudiar español son muy variadas, pero el hecho de considerar que es una lengua más fácil que las otras que se imparten parece ser el motivo principal de su elección, seguido del hecho de que es una de las lenguas más habladas o que tenían curiosidad por conocer la lengua debido a que ya habían estudiado las otras con anterioridad.

Antes de finalizar, señalar que no se ha encontrado un motivo exclusivo para la elección por parte de los alumnos del español como lengua extranjera. Las motivaciones son numerosas y variadas. Al ser alumnos de diferentes carreras sus motivaciones también difieren entre sí, aunque se denotan intereses específicos comunes que motivan su elección. Sin embargo, se ha de tener muy presente el contexto de la investigación, pues el hecho de estar cerca de España, y siendo la mayoría de los alumnos procedentes de Portalegre, ciudad cercana a las provincias de Cáceres y Badajoz, ven en este país una futura salida laboral. El alumnado ve en el país vecino una posibilidad de futuro y esto nos acerca a la idea del «valor económicon de la lengua, es decir, parece ser que la lengua es interesante en la medida en que el país en el que se habla tiene poder económico y ofrece posibilidades laborales, algo que puede cambiar a lo largo del tiempo cuando se pierda dicho atractivo. Se hace patente, una vez más, la necesidad de apostar por el español como lengua de ciencia y cultura, ya que el posible crecimiento de la crisis económica en los países de habla hispana puede perjudicar el crecimiento futuro de la lengua.

Por último, pensamos que este estudio, aun siendo de carácter regional, se podría ampliar a otras zonas de Portugal, principalmente a aquellas más fronterizas como Guarda o Braganza. A su vez, podría ser interesante realizar un estudio comparativo de las motivaciones de los alumnos de español en diferentes regiones del país, puesto que en este caso el hecho de estar cerca de España, y todo lo que ello implica, ha tenido un peso decisivo en la elección de los alumnos. Del mismo modo, también sería interesante analizar las motivaciones de los alumnos de otros niveles educativos. 
C. SANTíÑO GRELO, A. M. RODRÍGUEZ-GARCÍA Y M. A. HINOJO LUCENA

ESTUDIO DE LA ELECCIÓN DEL ESPAÑOL COMO LENGUA EXTRANJERA EN LA ESCUELA SUPERIOR DE PORTALEGRE (PORTUGAL)

\section{REFERENCIAS BIBLIOGRÁFICAS}

Almeida, J. C. P. (1995). Uma metodologia específica para o ensino de línguas próximas? Campinas: Pontes.

Aznar, I.; Cáceres, M. P. e Hinojo, F. J. (2008). La diversidad de estilos de aprendizaje y sus principales implicaciones psicopedagógicas en el procesamiento de información multimodal y multilingüe. En R. Arroyo (Ed.). La comunicación escrita: cognición, multilingüismo y tecnologías (pp. 303-319). Granada: Nativola.

Baralo, M. (1999). La adquisición del español como lengua extranjera. Madrid: Arco Libros.

Bernardo, A.; Amérigo, M. y García, J. A. (2014). Acquisition of Spanish as a Foreign Language through the socioeducational model a cross-cultural analysis. Journal of Language and Social Psychology, 33 (5), 500-516.

Campos, M. J.; Sola, T. y Aznar, I. (2014). Estudo das metodologias e estratégias do Programa de Português dos Cursos Profissionais de nível secundário: o caso do concelho de Almada. REICE: Revista Electrónica Iberoamericana sobre Calidad, Eficacia y Cambio en Educación, 12 (2), 29-41.

Catalán, D. (1989). Las lenguas circunvecinas del castellano. Madrid: Paraninfo.

Dittami, M. (1998). Español e italiano: peligroso parentesco románico y falsa amistad léxica. En A. Bueno y J. García (Coords.). La traducción: de la teoría a la práctica. Valladolid: Servicio de Apoyo a la Enseñanza. Universidad de Valladolid.

Flores-Ferrán, N. y Lovejoy, K. (2015). An examination of mitigating devices in the argument interactions of L2 Spanish learners. Journal of Pragmatics, 76 (1), 67-86.

Franco, P.; Pino, R. y Rodríguez, B. (2010). Tipología y frecuencia del uso de estrategias en el aprendizaje del inglés como lengua extranjera. Enseñanza \& Teaching, 27 (2), 171-191.

García, P. N. (2014). Methodological developments in teaching Spanish as a second and foreign language. Hispania-A Journal devoted to the teaching of Spanish and Portuguese, 97 (3), 529-530.

Gómez, A. (2011). Sobre la proximidad entre el portugués y el español. Revisión y análisis de datos: los falsos amigos y los préstamos. En Actas del IV Congreso sobre la Enseñanza del Español en Portugal (pp. 80-98). Universidad de Évora. Descargado el 6 de diciembre de 2015. http://redined.mecd.gob.es/xmlui/handle/11162/98821.

Martín, J. M. (2012). La enseñanza del español en Lisboa. Tejuelo, 14, 137-143.

Menéndez, R. (1980). Orígenes del español. Estado lingüístico de la península ibérica hasta el siglo XI. Madrid: Espasa-Calpe.

Mira, A. R. (2012). La enseñanza de la lengua española en Portugal. Tejuelo, 14, 86-108.

Nakatsukasa, K. y Loewen, S. (2015). A teacher's first language use in in form-focused episodes in Spanish as a foreign language classroom. Language Teaching Research, 19 (2), 133-149.

Pierce, J. y Cuadrado, G. (2011). Desarrollo de las competencias lingüísticas a través del Portfolio de Inglés Académico y Profesional. Enseñanza \& Teaching: Revista Interuniversitaria de Didáctica, 2 (29), 125-145.

Rabadán, M. (2014). Las actitudes hacia el español como lengua extranjera en el contexto universitario portugués. En E. Tobar y Mañas, M. (Coords.). El español como lengua extranjera en Portugal: retos de la enseñanza de lenguas cercanas (pp. 105-113). Madrid: Secretaría General Técnica.

Ricardo, A. (2012). La enseñanza de la lengua española en Portugal. Tejuelo, 14, 86-108.

Sánchez, A. (2014). Spanish as a foreign language in Europe: six centuries of teaching materials. Language \& History, 57 (1), 59-74. 
160 C. SANTÍÑO GRELO, A. M. RODRÍGUEZ-GARCÍA Y M. A. HINOJO LUCENA ESTUDIO DE LA ELECCIÓN DEL ESPAÑOL COMO LENGUA EXTRANJERA EN LA ESCUELA SUPERIOR DE PORTALEGRE (PORTUGAL)

Santíño, C. (2012). Estudio descriptivo de la didáctica y organización del español como lengua extranjera en la Escuela Superior de Educación de Portalegre (ESEP), Portugal (Tesis doctoral). Departamento de Didáctica y Organización Escolar. Universidad de Granada. Granada.

Santos, M. D. (2011). Las nuevas tecnologías en la clase de español como lengua extranjera. En Actas del IV Congreso sobre la Enseñanza del Español en Portugal (pp. 282-294). Universidad de Évora. Descargado el 3 de enero de 2016. http://redined.mecd.gob. es/xmlui/bitstream/handle/11162/99004/Las\%20nuevas\%20tecnolog\%C3\%ADas\%20 en $\% 201$ a $\% 20$ clase $\% 20 \mathrm{de} \% 20$ espa $\%$ C3\%B1ol\%20como\%20lengua\%20extranjera.pdf?sequence $=1$.

Sevillano, M. L. (2011). Didáctica en el núcleo de la pedagogía. Tendencias Pedagógicas, $18,7-32$.

Sola, T.; Hinojo, F. J. y Cáceres, M. ${ }^{a}$ P. (2010). Estudio de las dificultades en el dominio de la competencia fonológica del aprendizaje de la lectoescritura en el alumnado de $4 .^{\circ}$ nivel de Educación Primaria. Revista Española de Pedagogía, 68 (246), 333-361.

Sweet, H. (1964). The practical study of language: a guide for teachers and learners. Oxford: Oxford University Press.

Takeuchi, N. (1980). Um estudo de interferencia lexical. Curitiva. Dissertação (Mestrado em Letras)-UFPR.

Tobar, E. y Mañas, M. (Coords.) (2014). El español como lengua extranjera en Portugal: retos de la enseñanza de lenguas cercanas. Madrid: Secretaría General Técnica.

Vigón, S. (2004). La enseñanza de la gramática del español como lengua extranjera a lusófonos. En Actas del XV Congreso ASELE (pp. 903-914). Sevilla: Universidad de Sevilla. Descargado el 27 de noviembre de 2015. http://repositorium.sdum.uminho.pt/ handle/1822/6084?locale=es.

Vigón, S.; Humblé, P. y Vasconcellos, M. L. (2005). La enseñanza del español en los estudios de traducción en Portugal y Brasil. En Actas del II Congreso AIETI. Madrid: Universidad de Comillas (pp. 269-283). Descargado el 27 de noviembre de 2015. http://repositorium.sdum.uminho.pt/handle/1822/5968.

Vigón, S. y Saracho, M. (2008). Las Universidades, los politécnicos y los profesores de español en Portugal. En El profesor de español LE-L2: Actas del XIX Congreso Internacional de la Asociación para la Enseñanza del Español como Lengua Extranjera (ASELE) (pp. 779-772). Cáceres. Descargado el 16 de diciembre de 2015. http://cvc.cervantes.es/ Ensenanza/biblioteca_ele/asele/pdf/19/19_0779.pdf.

Vivanco, H. (2007). Unidad en la diversidad lingüística. La enseñanza del español como lengua extranjera y su certificación. En Actas del IV Congreso Internacional de la Lengua Española. Cartagena. Descargado el 2 de diciembre de 2015. http://congresosdelalengua.es/cartagena/ponencias/seccion_3/36/vivanco_hiram.htm. 